\title{
Development of a novel printed circuit board technology for inductive device applications
}

\author{
O. Dezuari, S.E. Gilbert, E. Belloy, M.A.M. Gijs * \\ Institute of Microsystems, Swiss Federal Institute of Technology (EPFL), CH-1015 Lausanne, Switzerland
}

Accepted 25 November 1998

\begin{abstract}
This paper describes the fabrication and characterisation of 2-dimensional inductive devices integrated inside printed circuit boards (PCB) and flex-foils. These devices basically are composed of three layers of which the outer layers bear the printed coil patterns and the inner layer is a high permeability ferromagnetic sheet core. Both magnetic metal and copper layers are patterned using standard lithographic techniques. Electroplated interconnections between the outer layers complete the windings. We have fabricated both transformers and fluxgate magnetic field sensing devices with a thickness of $200 \mu \mathrm{m}$ for the flex-foil devices and $600 \mu \mathrm{m}$ for the PCB-based devices. Lateral dimensions are approximately $1 \mathrm{~cm}$. We realise relatively high inductances of $1-10 \mu \mathrm{H}$ at a frequency of 1 $\mathrm{kHz}$ for the transformers and a magnetic field detection limit of $43 \mu \mathrm{T}$ at $20 \mathrm{kHz}$ for the fluxgate devices. (C) 1999 Elsevier Science S.A. All rights reserved.
\end{abstract}

Keywords: Planar transformers; Fluxgate sensors; Thin inductive devices; PCB technology; Flex-foil technology

\section{Introduction}

Inductive and transformer-like devices are at the heart of numerous sensing and actuating applications. With the increasing trend of miniaturisation of electronic devices, often, the inductive components are the determining factor limiting further size reduction. Both for the realisation of high inductance transformers [1,2] and for high resolution magnetic sensing devices, based on inductive measurement principles like fluxgate sensors [3-8], the availability of a high permeability and low hysteresis ferromagnetic core material is essential.

We have developed a new method for the fabrication of planar microtransformers [9] and fluxgate sensing devices [10] using a hybrid printed circuit board (PCB)/flexible foil technology. In this process, a lithographically structured amorphous magnetic foil core is laminated between thin planar coil windings on epoxy boards or flexible polyimide foils, bonded together using standard PCB and flex-foil assembly techniques. The magnetic core material has an extremely high relative magnetic permeability $\left(\mu_{\mathrm{r}}\right.$

\footnotetext{
* Corresponding author. DMT-IMS, EPFL BM (Ecublens) 1015 Lausanne, Switzerland. Tel.: +41-21-693-6734; Fax: +41-21-693-5950; E-mail: martin.gijs@epfl.ch
}

$=100000)$ and a fairly large thickness $(25 \mu \mathrm{m})$. These numbers are one or two orders of magnitude greater than the corresponding properties of films made using classical vacuum deposition methods. Consequently, for a given inductor size, the inductance value can be much higher than obtained before. Moreover, in the present technology, the core material can be selected from a variety of commercially available amorphous magnetic foils, amenable to lithographic patterning.

\section{Fabrication procedure}

\subsection{Choice of the magnetic core}

For purposes of obtaining a large magnetic flux in a small volume, a planar transformer requires a sheet core having a very high relative permeability $\mu_{\mathrm{r}}$. The latter is equally well important for the realisation of high sensitivity fluxgates. Among commercially available materials, the Vitrovac ${ }^{\circledR}$ magnetic metals [11] are amorphous, soft magnetic CoFeMo alloys, featuring very low coercivities and extremely high permeabilities at low frequencies. Moreover, they exhibit high mechanical strength and stress resistance, rendering them amenable to integration in mul- 


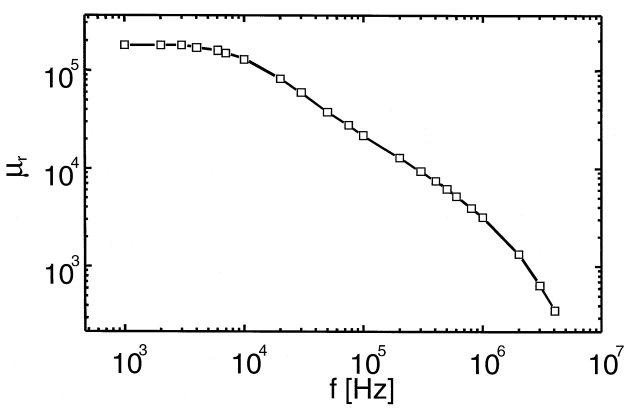

Fig. 1. Permeability vs. frequency of Vitrovac ${ }^{\circledR} 6025$.

tilayers fabrication techniques. For this work, Vitrovac ${ }^{\circledR}$ 6025 was used for the planar core. Its frequency-dependent permeability is reproduced in Fig. 1.

\subsection{Fabrication process}

The proposed structures were fabricated based on conventional PCB and flex-foil processes. Two copper-clad epoxy boards or polyimide foils were used as the outer layers. A liquid epoxy or acrylic adhesive was used to glue Vitrovac ${ }^{\circledR}$ foil on the interior non copper-bearing support, forming the magnetic core of the transformer.

Vitrovac ${ }^{\circledR}$ was patterned photolithographically to form the transformer cores. Coil patterns were printed onto the copper laminates using standard photolithographic methods. The conductor width was $100 \mu \mathrm{m}$, the thickness 35 $\mu \mathrm{m}$ and the distance between adjacent turns approximately $250 \mu \mathrm{m}$. Alternate half-windings were structured into each outer laminate layer, and corresponding top and bottom coil windings were connected by copper-filled vias. PCBbased structures were finally assembled and bonded by hot pressing with Prepreg ${ }^{\circledR}$ epoxy sheets, whereas for the flex-foil structure, we used acrylic adhesive layers. The

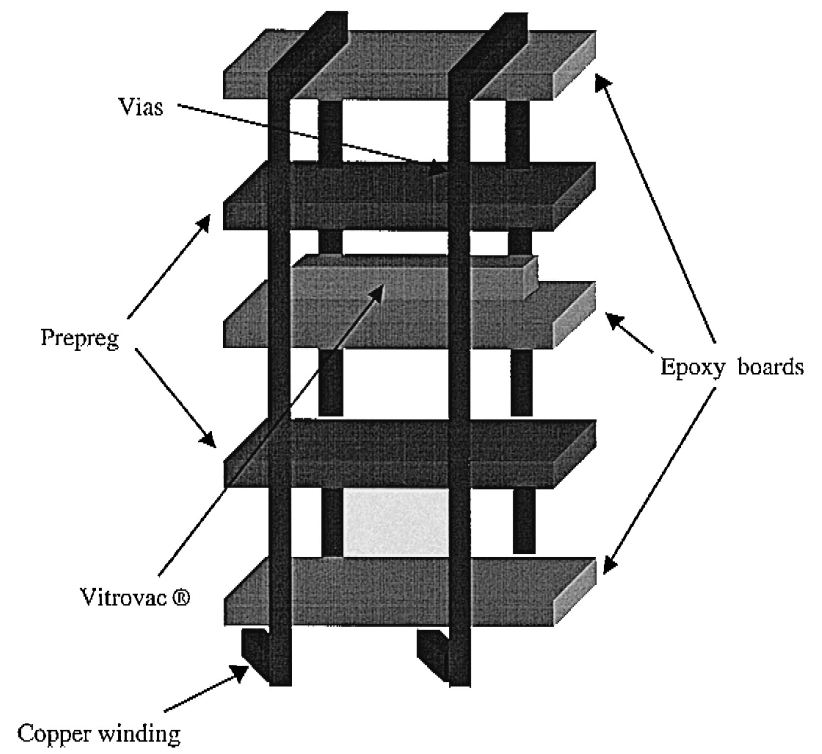

Fig. 2. Schematic diagram of inductive devices fabrication process.

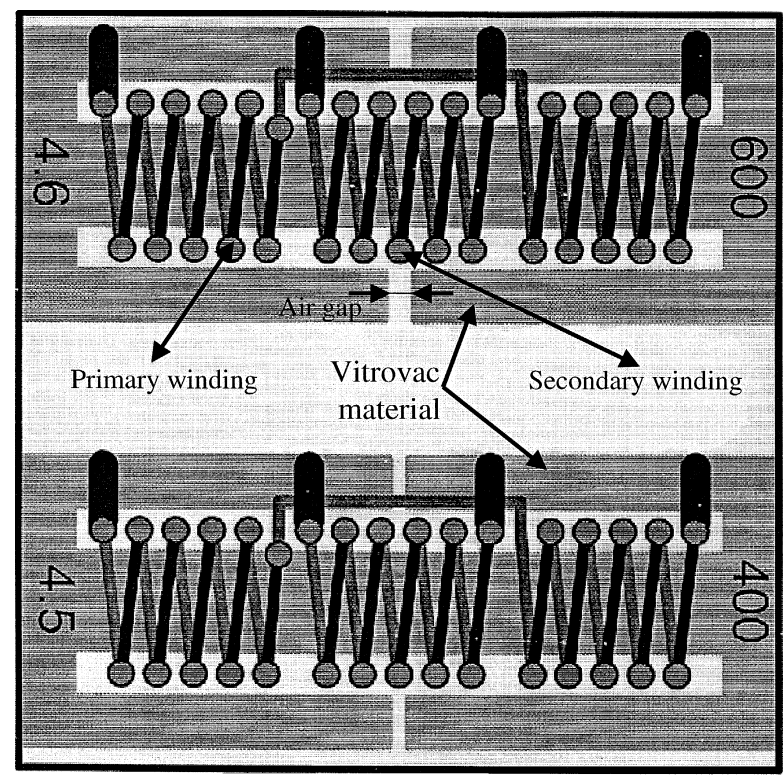

Fig. 3. Design diagram of two rectangular transformers showing air gaps in the magnetic material.

final PCB structure is schematised in Fig. 2 and is very analogous to the flex-foil-based structure.

\section{Design}

\subsection{Transformer devices}

Fig. 3 shows a design diagram for two transformers with rectangularly shaped magnetic core (E-core) whose

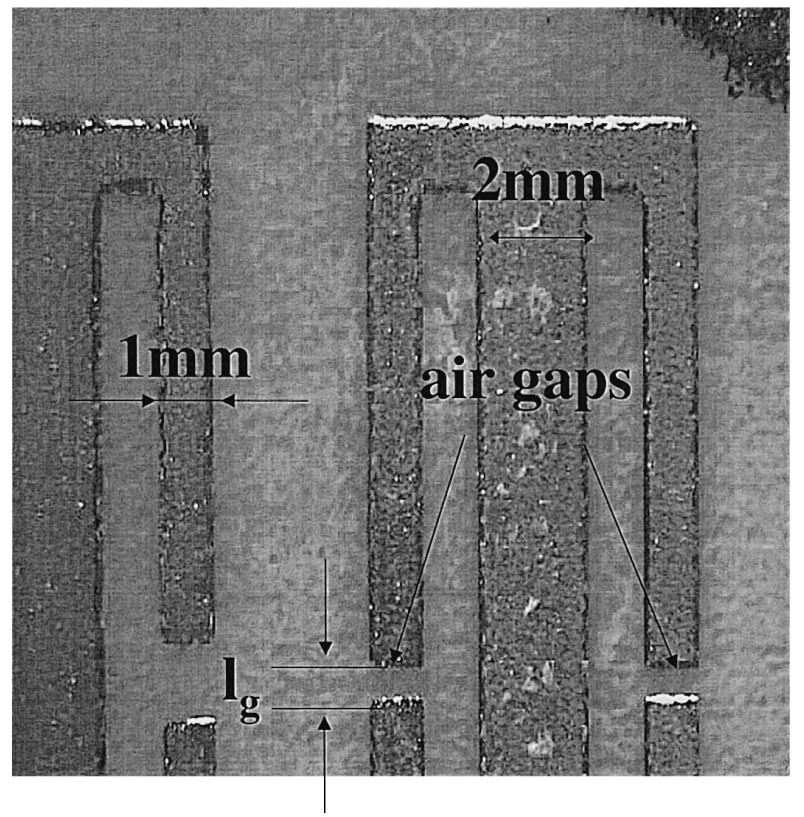

Fig. 4. Details of structured Vitrovac ${ }^{\circledR}$ E-cores, showing the two air gaps $l_{\mathrm{g}}$. 


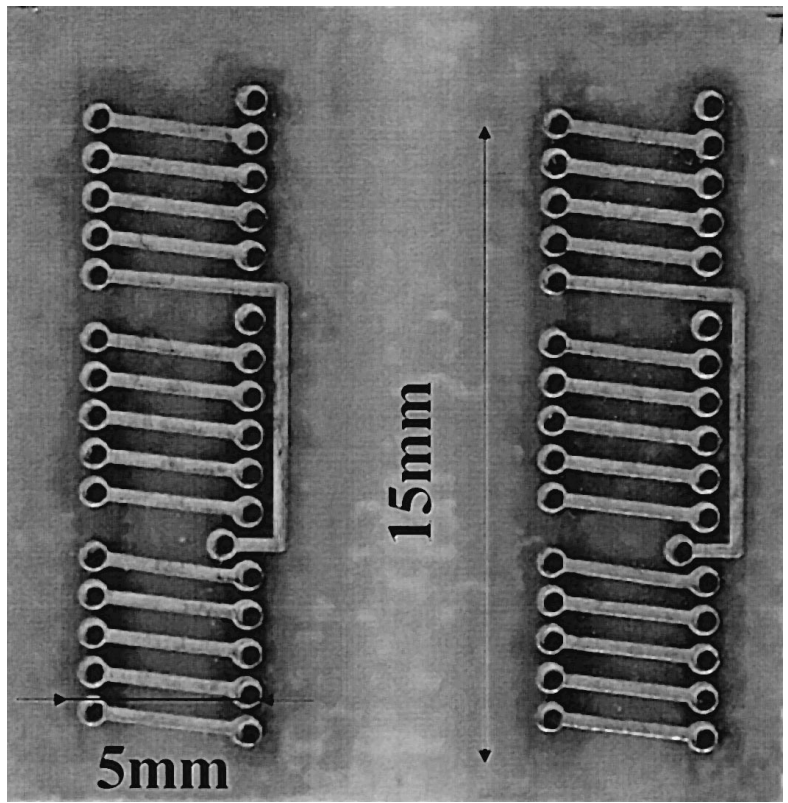

Fig. 5. Top view of finished transformers with E-cores.

primary windings have been grouped into two clusters of five, and are symmetrically positioned around the five secondary windings. All windings are around the inner magnetic part of the structure, while flux closure of the magnetic circuit is obtained via the two outer 'legs' of the magnetic core. Moreover, we have introduced air gaps in the outer two legs of the magnetic material. A series of E-core transformers with gap widths varying between 0 and $1000 \mu \mathrm{m}$ were fabricated to investigate how the

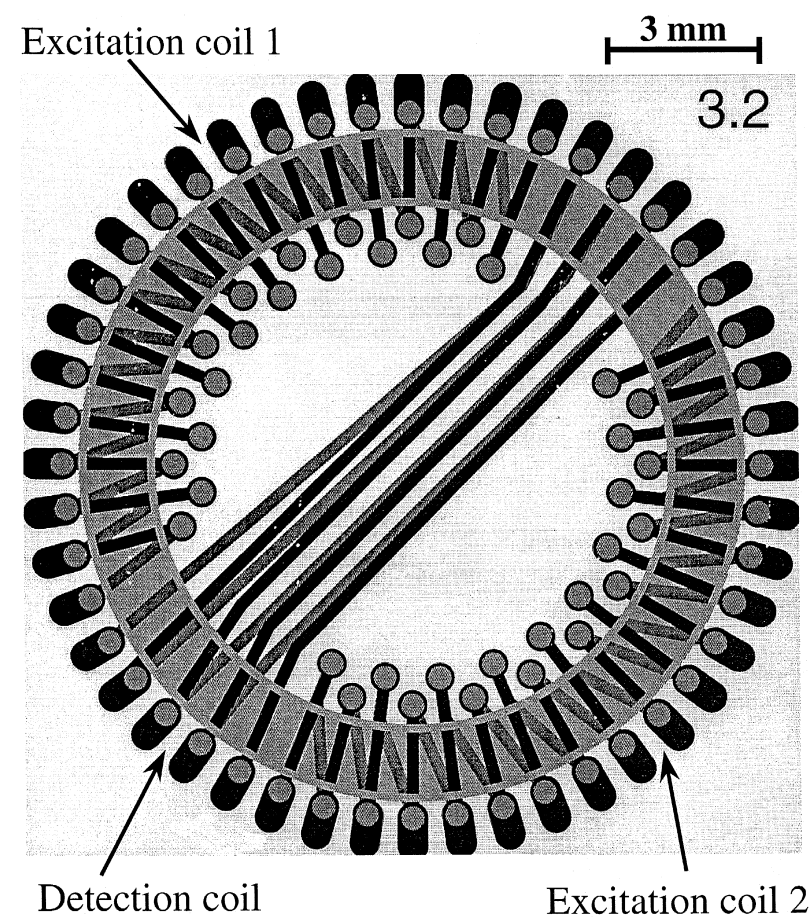

Fig. 6. Design diagram of a toroidal fluxgate sensor. inductance of such transformer behaves as a function of the width of the gap, as will be discussed below. Photographs of structured magnetic cores and of the complete transformers are displayed in Figs. 4 and 5.

\subsection{Fluxgate devices}

Fig. 6 shows a typical design diagram of the toroidal shaped fluxgate sensors. One should note that in this figure, the two excitation coils are positioned symmetrically at both sides of the detection coil. Each excitation coil has 18 windings while only four were used for the detection coil. Fig. 7a,b shows a finalised fluxgate device with at its interior a circular magnetic core realised in PCB or flex-foil material, respectively. The principle of a fluxgate is the external magnetic field-dependent periodic satu-
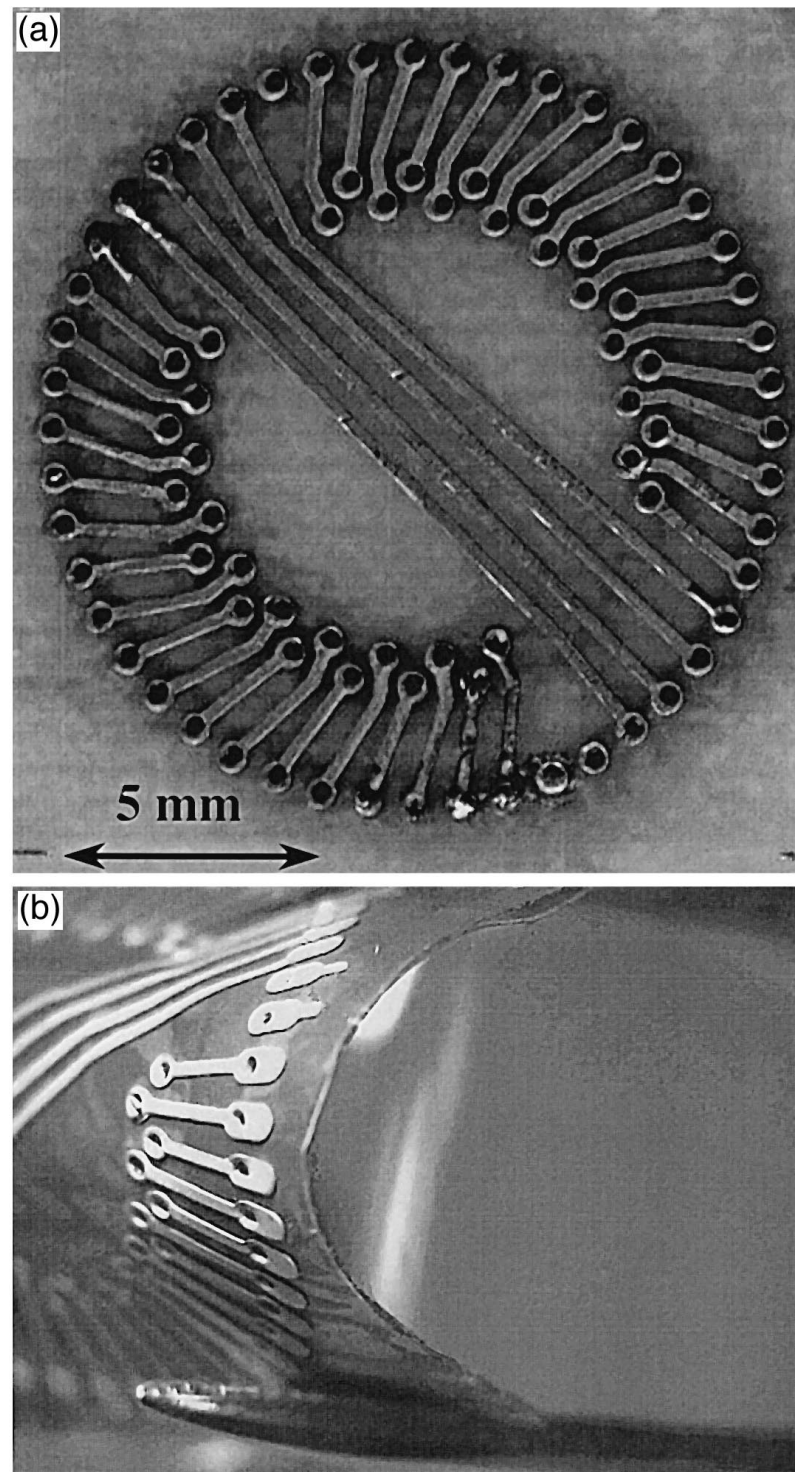

Fig. 7. (a) Top view of finished toroidal fluxgate sensor in PCB; (b) Toroidal fluxgate sensor realised with flex-foil technology. 


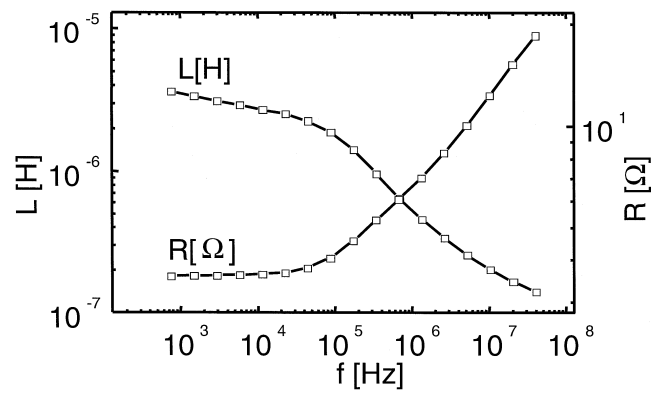

Fig. 8. Inductance and resistance as a function of frequency for a rectangular transformer without gap.

ration of a ferromagnetic material. Soft magnetic alloys like $\mathrm{Ni}_{80} \mathrm{Fe}_{20}$ (permalloy), having a small hysteresis and a large saturation magnetisation are well known for their use as core material. The combined action of the external field (to be measured) and excitation coils, driving the ferromagnetic core into saturation, leads to the generation of higher order harmonics of the fundamental excitation frequency in a detection coil surrounding the magnetic core. The second harmonic voltage amplitude $V_{2 \mathrm{f}}$ can be picked up from the detection signal using a lock-in technique and is proportional to the external field for a certain field range (typically $0.01-100 \mu \mathrm{T}$ ).

\section{Experimental results and discussion}

\subsection{Transformer measurements}

\subsubsection{Inductances measurements}

We show in Fig. 8 the frequency dependence of the primary self-inductance, $L$, and coil resistance, $R$, measured on a 10 turn primary rectangular transformer with an open secondary winding. The self inductance $L$ attains its highest values at low frequencies, and in this case a maximum of about $3.5 \mu \mathrm{H}$ is measured at $1 \mathrm{kHz}$. The inductance characteristic follows the intrinsic permeability dependence (Fig. 1) to some extent, but is also degraded by leakage flux. The rise in coil resistance starting at about $50 \mathrm{kHz}$ is primarily due to eddy current and magnetic core losses. Measurements of $L$ as a function of the number of

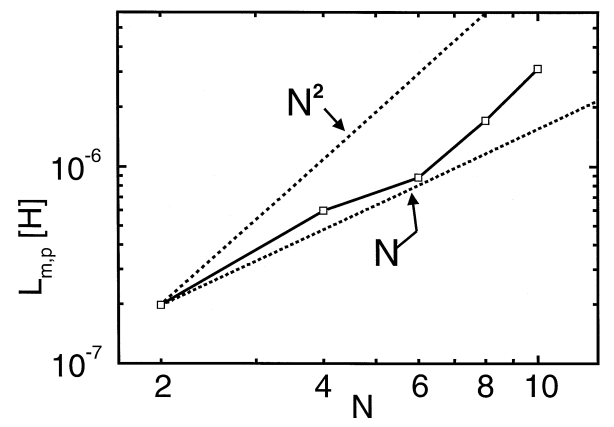

Fig. 9. Inductance vs. number of turns for a rectangular transformer.

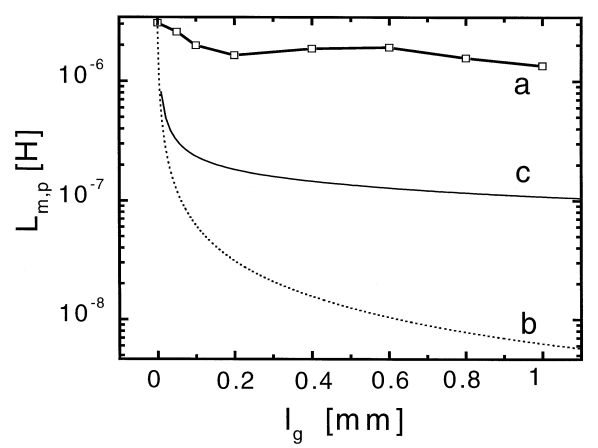

Fig. 10. Inductance as a function of gap $l_{\mathrm{g}}$ for a rectangular shaped core at $1 \mathrm{kHz}$ : Curve (a), measured values; curve (b), theoretical curve, (Eq. (1)); curve (c), (Eq. (1)) corrected by fringe factor $F$.

turns, $N$, is shown in the plot of Fig. 9. These measurements were made on a gapless rectangular transformer. The $\log -\log$ plot reveals a power law for $N$ of about 2 for a sufficient number of windings.

The effect of introducing air gaps in the outer legs of rectangular E-core type transformers is demonstrated in Fig. 10 (curve a). The inductance $L$ falls as the gap increases, thus following the classical behaviour of conventional transformers. However, $L$ maintains a rather constant value after the gap reaches $400 \mu \mathrm{m}$. To a first approximation, $L$ vs. the gap width, is given by:

$L=\frac{\mu_{\mathrm{o}} N^{2} A}{l_{\mathrm{g}}+\frac{l_{\mathrm{m}}}{\mu_{\mathrm{r}}}}$

where $L$ is the calculated inductance, $\mu_{\mathrm{o}}$ is the permeability of free space, $A$ is the cross-sectional area of the core legs, $N$, the number of windings, $l_{\mathrm{m}}$, the path length in the magnetic material, $\mu_{\mathrm{r}}$, the relative magnetic permeability and $l_{\mathrm{g}}$, the gap width. This equation is plotted as curve $\mathrm{b}$ in Fig. 10, based on an effective $\mu_{\mathrm{r}}$ of 16000 and $l_{\mathrm{m}}$ of $33.1 \mathrm{~mm}$. However, the experimental values are grossly underestimated by this theoretical curve, which predicts a more rapid fall-off of $L$ than is observed. Flux fringing

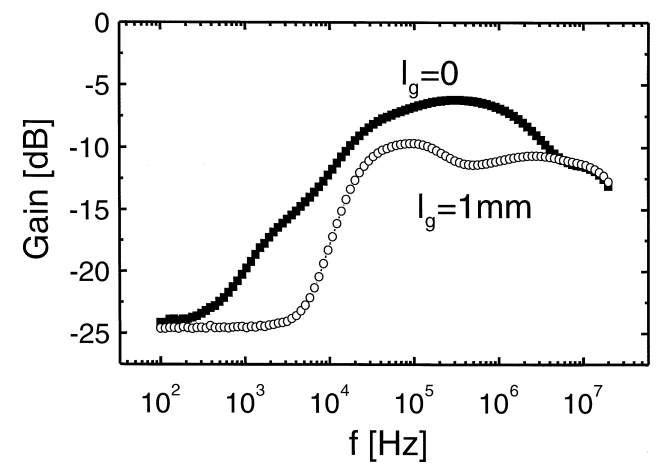

Fig. 11. Frequency dependence of transformation gain for rectangular microtransformers without gap (filled squares) and with air gap width $l_{\mathrm{g}}=1 \mathrm{~mm}$ (open circles). 
around the gaps, an effect which becomes more evident as the gap grows larger, can decrease the overall reluctance of the magnetic circuit, thereby offsetting the effect of the increasing gap. Its effect can be simulated by introducing a multiplicative correction factor, $F$, into Eq. (1), where
$F=1+l_{\mathrm{g}} / A^{1 / 2} \ln \left(2 G / l_{\mathrm{g}}\right)[9,10] . G$ is the vertical dimension of the core window ( $G=14 \mathrm{~mm}$ in our case).

We have plotted Eq. (1) with the correction as curve c in Fig. 10, demonstrating that there is better agreement with the experimental data. Thus, fringing flux at least
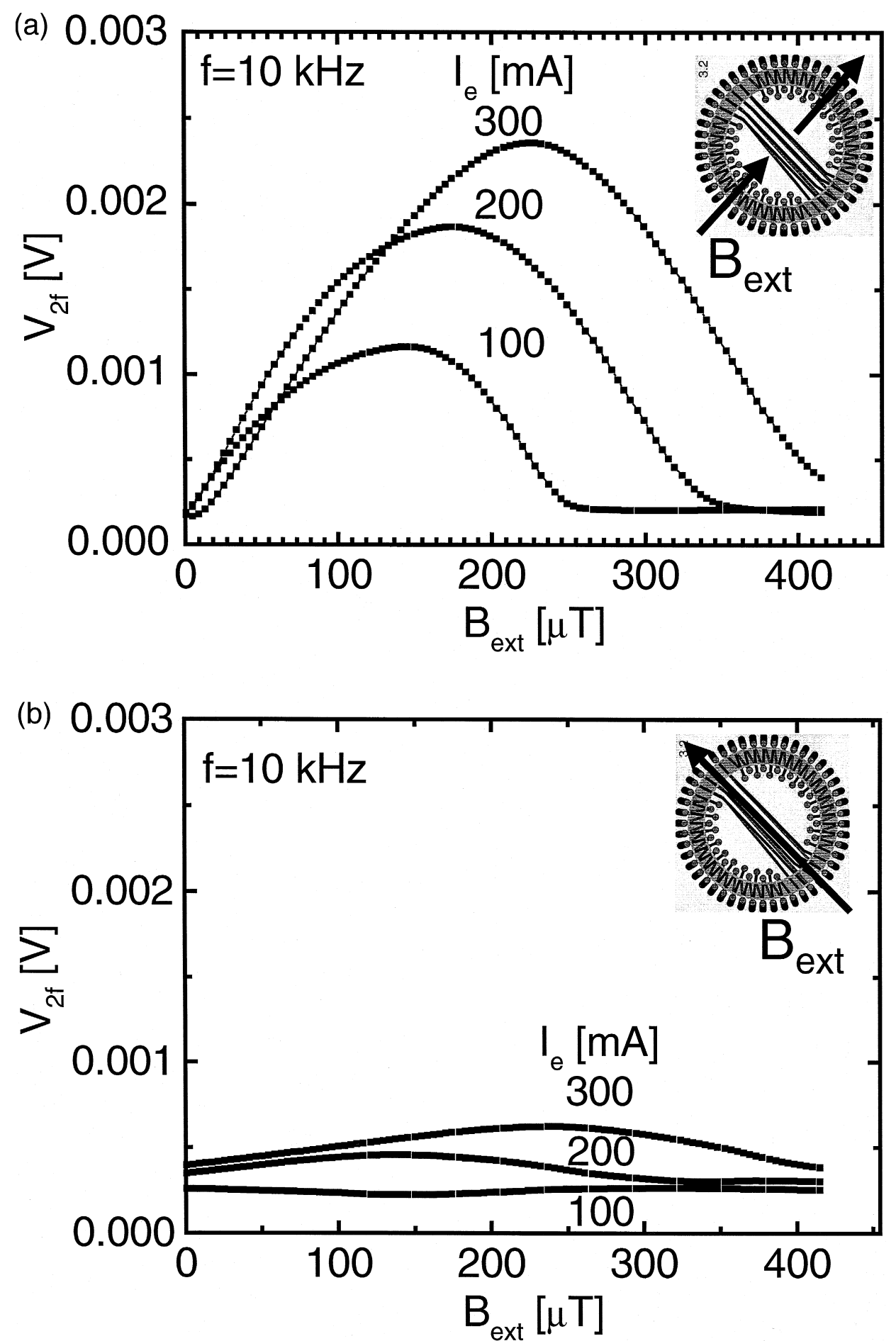

Fig. 12. Dependence on the orientation of the external field: (a) second harmonic voltage $V_{2 \mathrm{f}}$ of the detection coil of a toroidal fluxgate sensor as a function of $B_{\text {ext }}$ for various excitation currents $I_{\mathrm{e}}$ at $10 \mathrm{kHz}$; (b) second harmonic voltage $V_{2 \mathrm{f}}$ for the same fluxgate sensor and for the same choice of parameters as in (a), but for $B_{\text {ext }}$ applied parallel to the windings of the detection coil. 
partially accounts for the observed behaviour, but a secondary effect, that being the closure of the flux lines across the window space as the gap width becomes very large (1 $\mathrm{mm}$ ), may also play a significant role. This effect, which would be more important in microtransformers than in conventional ones, would reduce the mean length of the magnetic circuit $l_{\mathrm{m}}$. This point remains to be clarified, however.

\subsubsection{Transformer gain measurements}

Fig. 11 is a typical gain $\left(V_{\mathrm{sec}} / V_{\text {prim }}\right)$ plot for rectangular microtransformers with and without gap. The gain plots are measures of the real voltage transformation ratios and are indicative of the microtransformers' performance over a wide frequency range. For a rectangular transformer without a gap in a step-down configuration with a 2:1 turns ratio, the gain peaks at $-6.2 \mathrm{~dB}$ at approximately $300 \mathrm{kHz}$, corresponds to a voltage transformation ratio of 0.49 , or a $98 \%$ efficiency. The gain rests above $-7 \mathrm{~dB}$ (89\% efficiency) for frequencies ranging between $78 \mathrm{kHz}$ to $1 \mathrm{MHz}$ (see Fig. 11, upper curve). The gain curve for a rectangular transformer with a $1 \mathrm{~mm}$ gap is distinguished by a sharp rise starting at about $5 \mathrm{kHz}$, with a maximum of $-9.7 \mathrm{~dB}$ at $113 \mathrm{kHz}$ (Fig. 11, lower curve), corresponding to a voltage transformation ratio of 0.33 , or $66 \%$ transformation efficiency. Beyond this point, the gain is fairly flat over a very wide frequency range, and stays above -11 $\mathrm{dB}$ (56\% efficiency) at all frequencies between $30 \mathrm{kHz}$ to over $6 \mathrm{MHz}$. The lower transformer gain can be explained by a relatively larger importance of flux leaking away in the air.

\subsection{Fluxgate measurements}

\subsubsection{Dependence on the orientation of the external field}

Fig. 12a shows the second harmonic voltage amplitude $V_{2 \mathrm{f}}$ of the detection coil of a toroidal fluxgate sensor as a function of $B_{\text {ext }}$ for various excitation currents $I_{\mathrm{e}}$ at a frequency $f$ of $10 \mathrm{kHz}$. $B_{\text {ext }}$ is applied in-plane perpendicular to the detection coil, as shown schematically in the insert of Fig. 12a. For $I_{\mathrm{e}}=100 \mathrm{~mA}$, one notes a linear slope at small $B_{\text {ext }}$ and a maximum around $B_{\text {ext }}=150 \mu \mathrm{T}$. Above $250 \mu \mathrm{T}, V_{2 \mathrm{f}}$ approaches zero. For values of $I_{\mathrm{e}}$ between 200 and $300 \mathrm{~mA}$, the linear slope, the response maximum and the decrease of $V_{2 \mathrm{f}}$, all are shifted to higher $B_{\text {ext }}$. This graph clearly shows that, by judiciously choosing the excitation current $I_{\mathrm{e}}$, one can select the magnetic field sensitivity range and the linear part of the $V_{2 \mathrm{f}}-B_{\mathrm{ext}}$ curve.

Fig. $12 \mathrm{~b}$ shows the second harmonic voltage $V_{2 \mathrm{f}}$ for the same fluxgate sensor and for the same choice of parameters, except for the fact that $B_{\text {ext }}$ is applied parallel to the windings of the detection coil, as shown schematically in the insert. In this case, the external field does not directly cause an asymmetry within the detection coil. As a result, the response $V_{2 \mathrm{f}}$ has strongly decreased. Fig. 12a and b

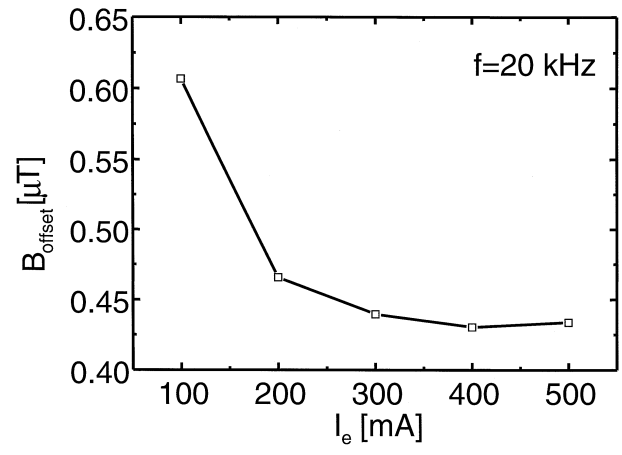

Fig. 13. Magnetic offset of the fluxgate sensor as a function of excitation current $I_{\mathrm{e}}$ at $20 \mathrm{kHz}$, for the rectangular fluxgate without gap.

both clearly show the potential of this fluxgate device for application as a magnetic compass.

\subsubsection{Magnetic offset of the fluxgate sensor}

To evaluate the minimum detection limit of our fluxgate sensing devices, we have performed measurements of the magnetic offset of the fluxgate sensor $B_{\text {offset }}$. We have measured the voltage $V_{2 \mathrm{f}}$ for a rectangular fluxgate, when cycling $B_{\text {ext }}$ from zero to saturation and back to zero. From the value of $V_{2 f}$ at this point, one can calculate the offset by using the fluxgate's measured sensitivity curves. The results of such procedure are shown in Fig. 13 as a function of excitation current $I_{\mathrm{e}}$ at $20 \mathrm{kHz}$. One observes a decreasing $B_{\text {offset }}$, i.e., an improved minimum field detection limit, with increasing $I_{\mathrm{e}}$. The reason for this behaviour has to be sought in the better defined magnetic saturation state at high applied $I_{\mathrm{e}}$ (smaller width of magnetic hysteresis curve at high $I_{\mathrm{e}}$ ). The observed values of $B_{\text {offset }}$ for our fluxgate sensor clearly are well below the value of the earth's magnetic field, enabling application of our devices for example as a magnetic compass.

\section{Conclusion}

In this paper, we have presented a new technology based on a low cost PCB or flexible foil process. The use of an amorphous metal foil as a magnetic core permits the facile development of high performance inductive devices. In comparison with existing technologies, this new process virtually eliminates the need for time consuming and expensive thin film deposition techniques, while retaining compatibility with standard electronic packaging schemes.

\section{Acknowledgements}

The authors would like to tank Mr. Laurent Chiesi and Mr. Pavel Kejik of the Institute of Microsystems for their assistance in the fluxgate measurements and Mr. Marc Hermanjat and Mr. Philippe Voessler of the department of 
electrical engineering at EPFL for their help in the process conception.

\section{References}

[1] K. Yamasawa, K. Maruyama, I. Hirohama, P. Biringer, High frequency operation of a planar-type microtransformer and its application to multilayered switching regulators, IEEE Trans. Mag. 26 (1990) 1204-1209.

[2] H. Tsujimoto, T. Koiso, Characteristic in the $0.01 \mathrm{GHz}-2.6 \mathrm{GHz}$ range of film transformer with coils on both sides of flexible polyamide film, IEEE Trans. Mag. 32 (1996) 4980-4982.

[3] F. Primdahl, The fluxgate magnetometer, J. Phys. E Sci. Instr. 12 (1979) 241-253.

[4] P. Ripka, Review of magnetic fluxgate sensor, Sensors Actuators A 33 (1992) 129-134.

[5] N. Pollock, Electronic compass using a fluxgate sensor, Wireless World (1982) 49-54.

[6] S. Kawahito, H. Satoh, M. Sutoh, Y. Tadokoro, High-resolution micro-fluxgate sensing elements using closely coupled coil structures, Sensors Actuators A 54 (1996) 612-617.

[7] R. Gottfried-Gottfried, W. Budde, R. Jähne, H. Kück, B. Sauer, S. Ulbricht, U. Wende, A miniaturised magnetic-field sensor system consisting of a planar fluxgate sensor and a CMOS readout circuitry, Sensors Actuators A 54 (1996) 443-447.

[8] T. Seitz, Fluxgate sensor in planar microtechnology, Sensors Actuators A 21-23 (1990) 799-802.

[9] O. Dezuari, S.E. Gilbert, E. Belloy, M.A.M. Gijs, A new hybrid technology for planar microtransformer fabrication, Sensors Actuators A 71/3 (1998) 198-207.

[10] O. Dezuari, E. Belloy, S.E. Gilbert, M.A.M. Gijs, A new hybrid technology for planar fluxgate sensor fabrication; IEEE Trans. Mag. 1995 (submitted for publication).

[11] R. Boll, Soft Magnetic Materials, Hanau, Vacuumschmeltze, 1993.

Olivier Dezuari was born in Lausanne, Switzerland in 1972. He received his diploma in microengineering from the Swiss Institute of Technology, EPF Lausanne in 1997. His diploma project was the simulation, fabrication and characterisation of a capacitive microphone for photoacoustic detection. He joined in September 1997 the Institute of Microsystems as research assistant in the field of developing new technologies for the fabrication of inductive components, in particular flexible components.
Scott E. Gilbert received his $\mathrm{PhD}$ in physical chemistry from the University of California, Santa Barbara in 1993 after completing a thesis on semiconductor surface electrochemistry. He then came immediately at the Swiss Federal Institute of Technology in Lausanne (EPFL) to postdoc at the Institute of Physical Chemistry for work on nanostructured liquid junction photovoltaic devices. Afterwards, he moved to the Department of Physics where his interests expanded to explore novel methods for ultra-thin metallic film and magnetic nanowire electrodeposition and electrical contacting methods. He is now with Prof. Gijs in the Institute of Microsystems as project leader for the development of new technologies for miniature inductive devices and electrochemical micromachining.

Eric Belloy was born in Geneva, Switzerland in 1972. He received his diploma in microengineering in 1997 from the Swiss Federal Institute of Technology of Lausanne (EPFL). His diploma work was the study, realisation and characterisation of microtips on $\mathrm{Si}$ and glass substrates, in use for the detection of the electrical activity of nerves cells. Since April 1997, he has been working as research assistant in the Institute of Microsystems at EPFL. He has first developed the technology process for a new magnetic sensor based on the Hall effect. Then, he has developed a new technology based on flex-foil patterning and assembling, in order to realise magnetic devices. His actual research area is on the dry-etching by micro-sandblasting.

Martin A.M. Gijs received his degree in physics in 1981 from the Katholieke Universiteit Leuven, Belgium and his $\mathrm{PhD}$ degree in physics at the same university in 1986. He joined the Philips Research Laboratories in Eindhoven, Netherlands in 1987. Subsequently, he has worked there on micro- and nano-fabrication processes of high critical temperature superconducting Josephson and tunnel junctions, the microfabrication of microstructures in magnetic multilayers showing the giant magnetoresistance effect, the design and realisation of miniaturised motors for hard disk applications and the design and realisation of planar transformers for miniaturised power applications. He joined the Swiss Federal Institute of Technology Lausanne (Ecole Polytechnique Fédérale de Lausanne) in 1997 as a professor in the Institute of Microsystems of the Microengineering Department, where he is responsible for the Microsystems Technology group. His main interests are in developing technologies for novel inductive-type devices, new microfabrication technologies for microsystems fabrication in general and the development and use of microsystems technologies for biomedical applications in particular. 